\title{
Nontraumatic Spontaneous Bilateral Basal Ganglia Hemorrhage: A Rare Case Report
}

\author{
Tariq A. Shaheed ${ }^{1}$, Nicholas Glover ${ }^{2}$, Safwan Alboiny ${ }^{3}$ \\ 1. Hospital Medicine / Internal Medicine, UC Davis Health, Lodi, USA 2. Graduate Medical Education / Emergency \\ Medicine, Desert Regional Medical Center, Palm Springs, USA 3. Neurology, Kaiser Permanente, Roseville, USA
}

Corresponding author: Tariq A. Shaheed, tariq.shaheed09@gmail.com

\begin{abstract}
A 62-year-old man with a past medical history of uncontrolled hypertension, tobacco abuse, and type 2 diabetes mellitus (DM) presented to the emergency department due to worsening confusion over the last 24 hours as reported by a friend. A CT brain without contrast was obtained, which demonstrated a bilateral intracerebral hemorrhage (ICH). Spontaneous bilateral intracerebral hemorrhage is an exceedingly rare condition with only 30-40 reported cases. This patient had a non-traumatic ICH, without focal neurological deficits on presentation. The patient had no complications while hospitalized despite the imaging findings. Clinicians should keep a broad differential similar to causes of spontaneous non-traumatic unilateral ICH, including uncontrolled hypertension, tumor mass, coagulopathies, and vasculopathies. Although brain CT is the most appropriate study in the acute setting, MRI is the gold standard for definitive diagnosis and should be performed urgently to further characterize the lesions. Clinicians should be aware of non-traumatic ICH complications, which include aspiration pneumonia, quadriparesis, hemiparesis, and recurrent stroke. Management is supportive mainly by reducing risk factors for complications, including blood pressure control, aspiration precautions, reversing coagulopathies, frequent neurological checks, and consultation with multiple disciplines such as neurosurgery or neurointerventional radiology.
\end{abstract}

Categories: Internal Medicine, Neurology, Miscellaneous

Keywords: intracerebral hemorrhage (ich), neurology, intracranial bleed, mri, head ct

\section{Introduction}

A spontaneous unilateral intracerebral hemorrhage (ICH) is a well documented medical condition; however, a spontaneous bilateral ICH is exceedingly rare, with only 30-40 reported cases [1]. The rare incidence of this disease process makes this topic specifically interesting. We discuss a curious case of a man who suffered a nontraumatic spontaneous bilateral ganglia ICH captured on medical imagery and is followed in our outpatient practice.

Review began 09/29/2020 Review ended 10/23/2020 Published 11/02/2020

\section{Copyright 2020}

Shaheed et al. This is an open access article distributed under the terms of the Creative Commons Attribution License CC-BY 4.0., which permits unrestricted use, distribution, and reproduction in any medium, provided the original author and source are credited.

\section{Case Presentation}

A 62-year-old man with a reported past medical history of uncontrolled hypertension was seen at our hospital emergency department due to worsening confusion over the last 24 hours with an acute exacerbation of confusion, witnessed by friend 30 minutes prior to the initial evaluation. Upon arrival, he had no specific complaints. He was on a daily $81 \mathrm{mg}$ aspirin and multiple blood pressure medications. He denied any other past medical or surgical history. He had no family history of diabetes; he had no history of coagulopathy but was unsure about any family history of any neurological disease or cancer. Social history was significant for smoking two cigarettes a day for 15 years but quit 10 years ago. On exam two hours after initial emergency department evaluation, he was alert and oriented to person, place, time, and event, but did elicit some confusion and had a Glasgow Coma Scale (GCS) of 14 (eye-opening - 4, verbal response - 4, motor response - 6). Cranial nerves II-XII were grossly intact, yet horizontal gaze was noted to be mildly abnormal. The remainder of his neurological exam was unremarkable with pupils equal, round, and reactive to light; sensation to light touch intact to upper and lower extremities; 5/5 grip strength as well as in the upper and lower extremities; deep tendon reflexes (DTRs) 2+/4 in upper and lower extremities bilaterally; no dysdiadochokinesia appreciated; and no truncal ataxia. The heart was found to be at a regular rate but bradycardic. No heart murmur was noted, and blood pressure was not elevated.

STAT computed tomography (CT) brain without contrast showed parenchymal hemorrhage of the right basal ganglia and intraparenchymal hemorrhage of the left lentiform nucleus (Figure 1). Comprehensive metabolic panel (CMP), complete blood count (CBC), and lipid panel were all within normal limits, except HDL of 25 $\mathrm{mg} / \mathrm{dL}$. A urine drug screen was negative. EKG demonstrated bradycardia at $55 \mathrm{bpm}$, but otherwise a normal EKG. A portable chest X-ray showed no acute cardiopulmonary disease. 


\section{Cureus}

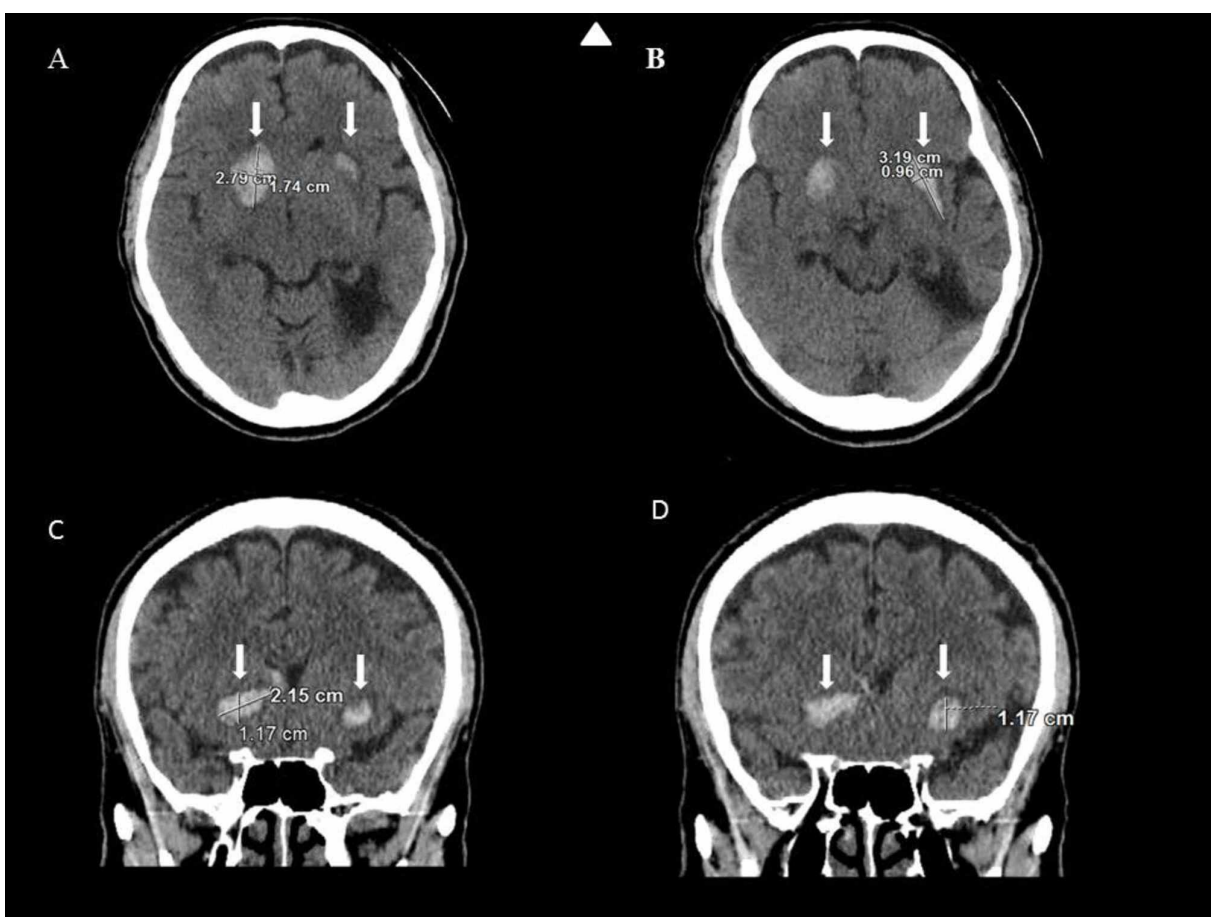

\section{FIGURE 1: Brain CT images}

Brain CT shows parenchymal hemorrhage of the right basal ganglia, measuring up to $2.8 \mathrm{~cm}$, with extension into the frontal horn of the right lateral ventricle. The same brain CT shows intraparenchymal hemorrhage of the left lentiform nucleus as well as chronic encephalomalacia of the left temporal lobe.

Arrowhead indicates top. A small arrow indicates areas of interest, which are hyperattenuation on noncontrast $\mathrm{CT}$ study consistent with $\mathrm{ICH}$.

A: CT WO transverse; hyperattenuation measures $2.79 \mathrm{~cm} \times 1.74 \mathrm{~cm}$

B: CT WO transverse; hyperattenuation measures $3.19 \mathrm{~cm} \times 0.96 \mathrm{~cm}$

C: CT WO coronal; hyperattenuation measures $2.15 \mathrm{~cm} \times 1.17 \mathrm{~cm}$

D: CT WO coronal; hyperattenuation measures $1.17 \mathrm{~cm}$

$\mathrm{ICH}$ - intracerebral hemorrhage; WO - without contrast

The patient was admitted to the ICU overnight and recovered an additional 48 hours on the telemetry floor. His hospital course was unremarkable except for one dose of hydralazine PRN, and his systolic blood pressure remained less than 160 . Neurosurgery was consulted, and no neurosurgical intervention was indicated. A magnetic resonance imaging (MRI) brain scan was obtained on the first day of admission demonstrated that hematoma was subacute in the bilateral basal ganglia, as seen on the initial scan (Figure 2) with no evidence of arteriovenous malformation (AVMs). MR neck angiogram with and without contrast showed evidence of mild atherosclerotic luminal narrowing of the right internal carotid origin and a hypoplastic left vertebral, which is usually developmental. An echocardiogram with a bubble study demonstrated a left ventricular ejection fraction greater than $60 \%$ with no wall abnormalities, no significant valvular regurgitation or vegetations, no thrombi identified, and no bubble contrast shunt from the interatrial septum. The patient was discharged after reaching the maximal benefit of hospital stay and is following in a hospital-associated outpatient clinic with the primary care provider (PCP) and neurology. 


\section{Cureus}

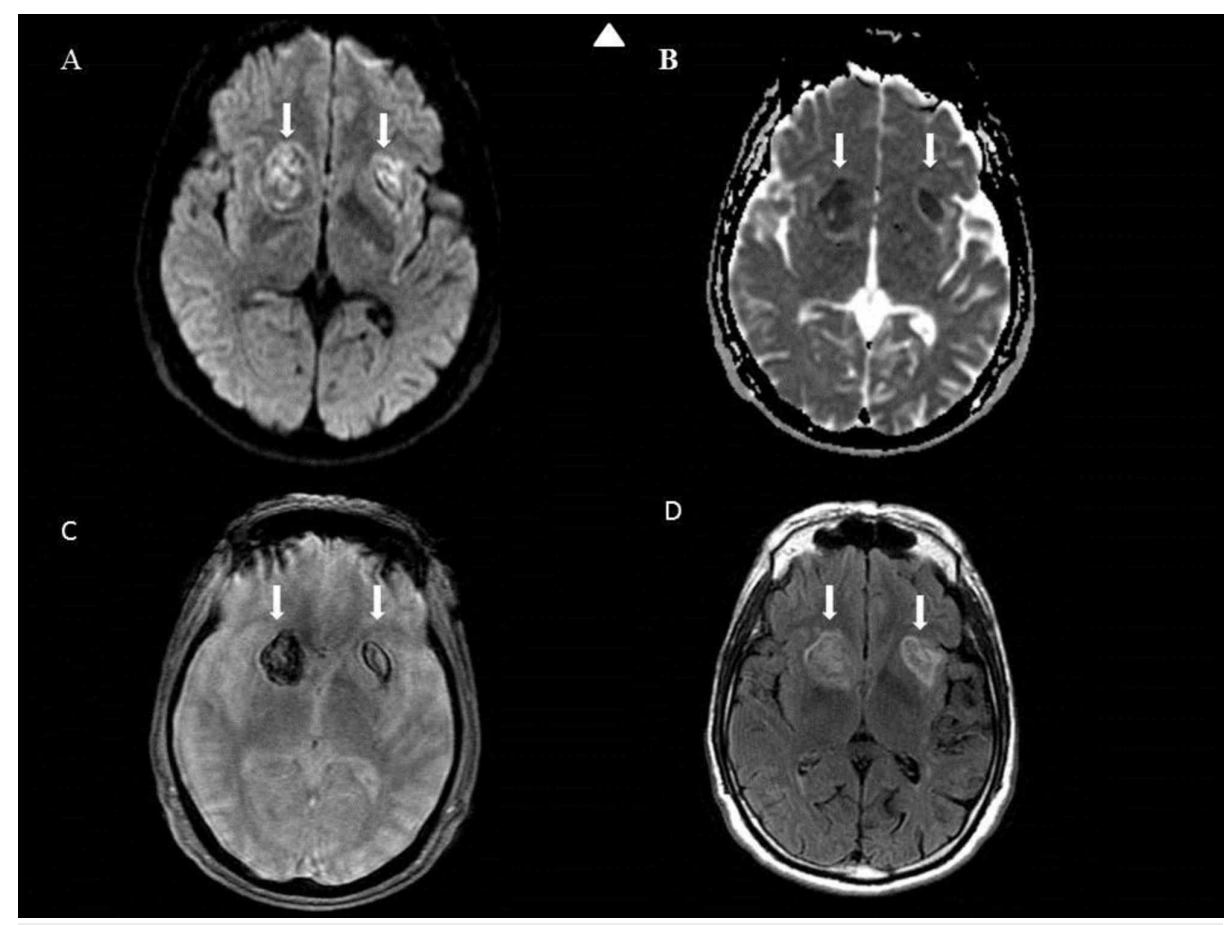

\section{FIGURE 2: MRI Brain}

Brain MRI demonstrates 1) no definite evidence of acute ischemic infarct; 2) subacute hematoma in the bilateral basal ganglia greater on the right with extension into the right frontal bone as on the previous CT; 3 ) small T1 and flair hyperintensity with possible slight enhancement at the posterior aspect of the left Sylvian fissure, possibly a small vascular lesion; 4) evidence of mild to moderate chronic ischemic foci in the white matter.

Arrowhead indicates top. Arrows indicate areas of interest on this MRI brain study with and without contrast. Correlates with clinical assessment of hypertensive subacute ICH in bilateral basal ganglia.
A: MRI WWO contrast DWI
B: MRI WWO contrast ADC
C: MRI WWO contrast GRE
D: MRI WWO contrast T2 flair

$\mathrm{ICH}$ - intracerebral hemorrhage; WWO - with and without contrast; DWE - diffusion-weighted images; ADC apparent diffusion coefficient; GRE - gradient recalled echo

\section{Discussion}

\section{Incidence}

Spontaneous unilateral intracerebral hemorrhage (ICH) is reported to be 24.6 per 100,000 person-year; however, a spontaneous bilateral ICH is exceedingly rare, with only 30-40 reported cases, and clinicians should be aware of the management, complications, and risk stratification recommendations. Of the previously reported cases of multiple spontaneous simultaneous ICH, $10.5 \%$ died, $10.5 \%$ was considered severely disabled, $2.6 \%$ was left in a vegetative state, $10.5 \%$ walked with a cane, and $5.2 \%$ had a good recovery [2].

\section{Differential diagnosis}

This patient presented with subtle changes in behavior and mental state but had no focal deficits on the exam, highlighting the importance for clinicians to consider spontaneous ICH in patients with mental status changes. The main causes of spontaneous ICH include hypertension, tumor mass, coagulopathies, and vasculopathies [2]. Risk factors such as a prior stroke, hypertension, and advanced age can cause both unilateral as well as bilateral intracerebral hemorrhage. An expanded differential diagnosis for bilateral abnormalities of the basal ganglia and thalamus includes cerebral amyloid angiopathy, which places patients at risk for multiple spontaneous intracerebral hemorrhages [2]. Even more rare differential diagnosis categories include poisoning, metabolic diseases, vascular diseases, degenerative diseases, inflammatory 


\section{Management}

Complications of a nontraumatic spontaneous bilateral basal ganglia ICH include aspiration pneumonia, quadriparesis, hemiparesis, and recurrent stroke [2]. Management is largely supportive by reducing risk factors for complications, including blood pressure control with a systolic blood pressure goal of 140 if there is no evidence of carotid stenosis and 160 if there is evidence of stenosis. Computed tomography angiography (CTA) neck or carotid doppler are the best studies to rule out carotid stenosis. Elevation of the head of the bed greater than 30 degrees assists in reducing the risk of aspiration. Early consultation with neurosurgery is paramount to evaluate any indications for any neurosurgical intervention [4].

\section{Conclusions}

Spontaneous bilateral ICH is a rare event and can present severely with quadriplegia or, as in this case, with mild neurological deficits. Advanced age, history of hypertension, and prior stroke are the most common risk factors for spontaneous bilateral ICH, but subsequent follow up is necessary to rule out more rare disease processes. Although the brain CT is the most appropriate initial study in the acute setting, MRI is the gold standard for definitive diagnosis and should be performed urgently to further characterize the lesions. CT angiography is a critical test to assess for vascular malformation, which could require intervention.

\section{Additional Information \\ Disclosures}

Human subjects: Consent was obtained by all participants in this study. Conflicts of interest: In compliance with the ICMJE uniform disclosure form, all authors declare the following: Payment/services info: All authors have declared that no financial support was received from any organization for the submitted work. Financial relationships: All authors have declared that they have no financial relationships at present or within the previous three years with any organizations that might have an interest in the submitted work. Other relationships: All authors have declared that there are no other relationships or activities that could appear to have influenced the submitted work.

\section{Acknowledgements}

We would like to thank our patient for sharing his case, as well as the Community Memorial Health System, for allowing us to take part in his care.

\section{References}

1. van Asch CJ, Luitse MJ, Rinkel GJ, van der Tweel I, Algra A, Klijn CJ: Incidence, case fatality, and functional outcome of intracerebral haemorrhage over time, accord- ing to age, sex, and ethnic origin: a systematic review and meta-analysis. Lancet Neurol. 2010, 9:167-176. 10.1016/S1474-4422(09)70340-0

2. Seo J-S, Nam T-K, Kwon J-T, Park Y-S: Multiple spontaneous simultaneous intracerebral hemorrhages. J Cerebrovasc Endovasc Neurosurg. 2014, 16:104-111. 10.7461/jcen.2014.16.2.104

3. Hegde AN, Mohan S, Lath N, Lim CC: Differential diagnosis for bilateral abnormalities of the basal ganglia and thalamus. Radiographics. 2011, 31:5-30. 10.1148/rg.311105041

4. Mulligan PR, Prajapati HJS, Martin LG, Patel TH: Vascular anomalies: classification, imaging characteristics and implications for interventional radiology treatment approaches. Brit J Radiol. 2014, 87:20130392. 10.1259/bjr.20130392 